ISSN 1855-3966 (printed edn.), ISSN 1855-3974 (electronic edn.)

ARS MATHEMATICA CONTEMPORANEA 18 (2020) 273-280

https://doi.org/10.26493/1855-3974.1957.a0f

(Also available at http://amc-journal.eu)

\title{
On the general position problem on Kneser graphs*
}

\author{
Balázs Patkós \\ Alfréd Rényi Institute of Mathematics, Budapest, H-1364, Hungary, and \\ Moscow Institute of Physics and Technology
}

Received 22 March 2019, accepted 9 March 2020, published online 20 October 2020

\begin{abstract}
In a graph $G$, a geodesic between two vertices $x$ and $y$ is a shortest path connecting $x$ to $y$. A subset $S$ of the vertices of $G$ is in general position if no vertex of $S$ lies on any geodesic between two other vertices of $S$. The size of a largest set of vertices in general position is the general position number that we denote by $g p(G)$. Recently, Ghorbani et al. proved that for any $k$ if $n \geq k^{3}-k^{2}+2 k-2$, then $g p\left(K n_{n, k}\right)=\left(\begin{array}{l}n-1 \\ k-1\end{array}\right)$, where $K n_{n, k}$ denotes the Kneser graph. We improve on their result and show that the same conclusion holds for $n \geq 2.5 k-0.5$ and this bound is best possible. Our main tools are a result on cross-intersecting families and a slight generalization of Bollobás's inequality on intersecting set pair systems.
\end{abstract}

Keywords: General position problem, Kneser graphs, intersection theorems.

Math. Subj. Class. (2020): 05D05, 05C35

\section{Introduction}

A recently studied extremal problem $[4,6,12]$ in graph theory is the following. In a graph $G$, a geodesic between two vertices $x$ and $y$ is a shortest path connecting $x$ to $y$. We say that a subset $S$ of the vertices of $G$ is in general position if no vertex of $S$ lies on any geodesic between two other vertices of $S$. The size of a largest set of vertices in general position

* The author would like to thank Sandi Klavžar and Gregor Rus for pointing out that sets of the star are not in general position if $n<2.5 k-0.5$. This was also pointed out later by both referees whom I thank for their thorough reading. I would like to thank Máté Vizer for showing me the relation of the families considered in the paper to qualitative independent partitions and Gábor Simonyi for providing me a short introduction to this topic.

${ }^{\dagger}$ Research supported by the National Research, Development and Innovation Office - NKFIH under the grants SNN 129364 and FK 132060. The author acknowledges the financial support from the Ministry of Education and Science of the Russian Federation in the framework of MegaGrant no. 075-15-2019-1926

E-mail address: patkos@ renyi.hu (Balázs Patkós) 
is the general position number which we denote by $g p(G)$. Our graph of interest in this paper is the Kneser graph $K n_{n, k}$ whose vertex is $\left(\begin{array}{c}{[n]} \\ k\end{array}\right)$, the set of all $k$-element subsets of the set $[n]=\{1,2, \ldots, n\}$ and two $k$-subsets $S$ and $T$ are joined by an edge if and only if $S \cap T=\emptyset$. Ghorbani et al. [10] determined $g p\left(K n_{n, 2}\right)$ and $g p\left(K n_{n, 3}\right)$ for all $n$ and showed that for any fixed $k$ if $n$ is large enough, then $g p\left(K n_{n, k}\right)=\left(\begin{array}{l}n-1 \\ k-1\end{array}\right)$ holds.

Theorem 1.1 ([10]). Let $n, k \geq 2$ be integers with $n \geq 3 k-1$. If for all $t$, where $2 \leq t \leq k$, the inequality $k^{t}\left(\begin{array}{l}n-t \\ k-t\end{array}\right)+t \leq\left(\begin{array}{l}n-1 \\ k-1\end{array}\right)$ holds, then gp $\left(K n_{n, k}\right)=\left(\begin{array}{l}n-1 \\ k-1\end{array}\right)$.

For fixed $k$ and $t=2$ the above inequality is satisfied when $n \geq k^{3}-k^{2}+2 k-1$ holds. We improve on this and the main result of this note is the following.

Theorem 1.2. If $n, k \geq 4$ are integers with $n \geq 2 k+1$, then gp $\left(K n_{n, k}\right) \leq\left(\begin{array}{l}n-1 \\ k-1\end{array}\right)$ holds. Moreover, if $n \geq 2.5 k-0.5$, then we have $g p\left(K n_{n, k}\right)=\left(\begin{array}{l}n-1 \\ k-1\end{array}\right)$, while if $2 k+1 \leq n<$ $2.5 k-0.5$, then $g p\left(K n_{n, k}\right)<\left(\begin{array}{l}n-1 \\ k-1\end{array}\right)$ holds.

The threshold $n \geq 2.5 k-0.5$ comes from the fact that $\operatorname{diam}\left(K n_{n, k}\right) \leq 3$ holds if and only if this inequality is satisfied. The proof of Theorem 1.1 uses the following general result of Anand et al. [2] that characterizes vertex subsets in general position.

Theorem 1.3 ([2]). If $G$ is a connected graph, then a subset $S$ of the vertices of $G$ is in general position if and only if all the components $S_{1}, S_{2}, \ldots, S_{h}$ of $G[S]$ are cliques in $G$ and

- for any $1 \leq i<j \leq h$ and $s_{i}, s_{i}^{\prime} \in S_{i}, s_{j}, s_{j}^{\prime} \in S_{j}$ we have $d\left(s_{i}, s_{j}\right)=d\left(s_{i}^{\prime}, s_{j}^{\prime}\right)=$ : $d\left(S_{i}, S_{j}\right)$ (where $d(x, y)$ denotes the distance of $x$ and $y$ in $G$ ),

- $d\left(S_{i}, S_{j}\right) \neq d\left(S_{i}, S_{l}\right)+d\left(S_{l}, S_{j}\right)$ for any $1 \leq i, j, l \leq h$.

In Kneser graphs a clique corresponds to a family $\mathcal{F} \subseteq\left(\begin{array}{c}{[n]} \\ k\end{array}\right)$ of pairwise disjoint sets. There is no edge between different components of any general position set $S$. It follows that if $\mathcal{F}_{1}, \mathcal{F}_{2}, \ldots, \mathcal{F}_{h}$ correspond to the components of $G[S]$, then for any $F_{i} \in \mathcal{F}_{i}$ and $F_{j} \in \mathcal{F}_{j}$ with $i \neq j$ we have $F_{i} \cap F_{j} \neq \emptyset$. Families with this property are called crossintersecting. So the upper bound in Theorem 1.2 will follow from the next result unless $n=2 k+1$ in which case we will need some further reasonings.

Theorem 1.4. Let $n \geq 2 k+2, k \geq 4$ and let $\mathcal{F}_{1}, \mathcal{F}_{2}, \ldots, \mathcal{F}_{h} \subseteq\left(\begin{array}{c}{[n]} \\ k\end{array}\right)$ such that

- $\mathcal{F}_{i} \cap \mathcal{F}_{j}=\emptyset$ for all $1 \leq i<j \leq h$,

- $F_{i} \cap F_{i}^{\prime}=\emptyset$ for all pairs of distinct sets $F_{i}, F_{i}^{\prime} \in \mathcal{F}_{i}$ for any $i=1,2, \ldots, h$,

- $F_{i} \cap F_{j} \neq \emptyset$ for any $1 \leq i<j \leq j$ and any $F_{i} \in \mathcal{F}_{i}, F_{j} \in \mathcal{F}_{j}$

hold. Then we have $\sum_{i=1}^{h}\left|\mathcal{F}_{i}\right| \leq\left(\begin{array}{l}n-1 \\ k-1\end{array}\right)$.

Note that the first condition cannot be omitted as otherwise we could repeat some families that consist of a single set.

The remainder of the paper is organized as follows: Section 2 contains the proof of Theorem 1.4 and in Section 3 we list some open problems along with some remarks. 


\section{Proofs}

Proof of Theorem 1.4. Let $\mathcal{F}_{1}, \mathcal{F}_{2}, \ldots, \mathcal{F}_{h} \subseteq\left(\begin{array}{c}{[n]} \\ k\end{array}\right)$ satisfy the conditions of the theorem. As the $\mathcal{F}_{i}$ 's are families of pairwise disjoint sets, each of them are of size at most $n / k$ and we may assume that $\left|\mathcal{F}_{1}\right| \leq\left|\mathcal{F}_{2}\right| \leq \cdots \leq\left|\mathcal{F}_{h}\right|=: t \leq n / k$. If $t=1$, then $\mathcal{F}=\cup_{i=1}^{h} \mathcal{F}_{i}$ form an intersecting family and therefore by the celebrated theorem of Erdős, Ko and Rado [5] we have $\sum_{i=1}^{h}\left|\mathcal{F}_{i}\right|=h \leq\left(\begin{array}{l}n-1 \\ k-1\end{array}\right)$.

Suppose next that $t \geq 2$ holds. Then we claim $h \leq\left(\begin{array}{c}n-1 \\ k-1\end{array}\right)-\left(\begin{array}{c}n-k-1 \\ k-1\end{array}\right)+1$. Indeed, let us fix one set $F_{i}$ from each $\mathcal{F}_{i}$ for $i=1,2, \ldots, h-1$ and two sets $F_{h}, F_{h}^{\prime} \in \mathcal{F}_{h}$. Hence if

- $\left|\cap_{i=1}^{h-1} F_{i}\right| \geq 2$, then $h-1 \leq\left(\begin{array}{l}n-2 \\ k-2\end{array}\right)<\left(\begin{array}{l}n-1 \\ k-1\end{array}\right)-\left(\begin{array}{c}n-k-1 \\ k-1\end{array}\right)$,

- $\cap_{i=1}^{h-1} F_{i}$ consists of a single element $x$, then either $F_{h}$ or $F_{h}^{\prime}$ cannot contain $x$ and as all $F_{i}$ 's meet both $F_{h}$ and $F_{h}^{\prime}$ we must have $h-1 \leq\left(\begin{array}{c}n-1 \\ k-1\end{array}\right)-\left(\begin{array}{c}n-k-1 \\ k-1\end{array}\right)$,

- $\cap_{i=1}^{h-1} F_{i}=\emptyset$, then $\left\{F_{1}, F_{2}, \ldots, F_{h-1}, F_{h}\right\}$ is intersecting with no common elements, and a result of Hilton and Milner [11] states that families with this property can have size at most $\left(\begin{array}{l}n-1 \\ k-1\end{array}\right)-\left(\begin{array}{c}n-k-1 \\ k-1\end{array}\right)+1$, so we obtain $h \leq\left(\begin{array}{l}n-1 \\ k-1\end{array}\right)-\left(\begin{array}{c}n-k-1 \\ k-1\end{array}\right)+1$.

Let $m_{i}$ denote the number of $j$ 's such that $\left|\mathcal{F}_{j}\right| \geq i$ holds. Then clearly we have

$$
\sum_{i=1}^{h}\left|\mathcal{F}_{i}\right|=h+\sum_{j=2}^{t} m_{j} \leq h+\left(\frac{n}{k}-1\right) m_{2} .
$$

To bound $m_{2}$ we apply Bollobás's famous inequality [3] that states that if $\left\{\left(A_{1}, B_{1}\right)\right\}_{i=1}^{l}$ are pairs of disjoint sets such that for any $1 \leq i \neq j \leq l$ we have $A_{i} \cap B_{j} \neq \emptyset$, then $\sum_{i=1}^{l} \frac{1}{\left(\begin{array}{c}\left|A_{i}\right|+\left|B_{i}\right| \\ \left|A_{i}\right|\end{array}\right)} \leq 1$ holds. For any $1 \leq i \leq m_{2}$ we can pick two sets $F_{i}, G_{i} \in \mathcal{F}_{h-m_{2}+i}$. Then we can define $2 m_{2}$ pairs $\left\{\left(A_{j}, B_{j}\right)\right\}_{j=1}^{2 m_{2}}$ such that for $1 \leq j \leq m_{2}$ we have $A_{j}=$ $F_{j}, B_{j}=G_{j}$ and $A_{2 m_{2}-j}=G_{j}, B_{2 m_{2}-j}=F_{j}$. As the $\mathcal{F}_{i}$ 's are cross-intersecting families of disjoint sets, therefore the pairs $\left\{\left(A_{j}, B_{j}\right)\right\}_{j=1}^{2 m_{2}}$ satisfy the conditions of Bollobás's inequality and we obtain $\frac{2 m_{2}}{\left(\begin{array}{c}2 k \\ k\end{array}\right)} \leq 1$ and thus $m_{2} \leq \frac{1}{2}\left(\begin{array}{c}2 k \\ k\end{array}\right)=\left(\begin{array}{c}2 k-1 \\ k-1\end{array}\right)$. Putting together (2.1) and the bounds on $h$ and $m_{2}$ we obtain

$$
\sum_{i=1}^{h}\left|\mathcal{F}_{i}\right| \leq\left(\begin{array}{c}
n-1 \\
k-1
\end{array}\right)-\left(\begin{array}{c}
n-k-1 \\
k-1
\end{array}\right)+1+\frac{n-k}{k}\left(\begin{array}{c}
2 k-1 \\
k-1
\end{array}\right)
$$

Therefore it is enough to prove $\left(\begin{array}{c}n-k-1 \\ k-1\end{array}\right)>\frac{n-k}{k}\left(\begin{array}{c}2 k-1 \\ k-1\end{array}\right)$. Observe that

$$
\frac{\left(\begin{array}{c}
n-k \\
k-1
\end{array}\right)}{\left(\begin{array}{c}
n-k-1 \\
k-1
\end{array}\right)}=\frac{n-k}{n-2 k+1} \geq \frac{n-k+1}{n-k}=\frac{\frac{n-k+1}{k}\left(\begin{array}{c}
2 k-1 \\
k-1
\end{array}\right)}{\frac{n-k}{k}\left(\begin{array}{c}
2 k-1 \\
k-1
\end{array}\right)},
$$

therefore if $\left(\begin{array}{c}n_{0}-k-1 \\ k-1\end{array}\right)>\frac{n_{0}-k}{k}\left(\begin{array}{c}2 k-1 \\ k-1\end{array}\right)$ holds for some $n_{0}$, then $\left(\begin{array}{c}n-k-1 \\ k-1\end{array}\right)>\frac{n-k}{k}\left(\begin{array}{c}2 k-1 \\ k-1\end{array}\right)$ holds for $n \geq n_{0}$. Putting $n_{0}=3 k+2$ the above inequality is equivalent to

$$
k \prod_{i=0}^{k-2}(2 k+1-i)>(2 k+2) \prod_{i=0}^{k-2}(2 k-1-i)
$$


which simpifies to

$$
k(2 k+1) 2 k>(2 k+2)(k+2)(k+1) .
$$

This holds for $k \geq 5$ and a similar calculation shows that if $k=4$, then the desired inequality holds if $n \geq 17=4 k+1$.

In all missing cases, except for $k=4, n=16$, we have $n<4 k$, therefore we have $m_{j}=0$ for all $j \geq 4$. So for the remaining pairs $n$ and $k$, we need to strengthen our bound on $m_{2}+m_{3}$. We will need the following lemma, a slight generalization of Bollobás's result.

Lemma 2.1. Let $\left\{A_{i}, B_{i}\right\}_{i=1}^{\alpha}$ and $\left\{A_{j}, B_{j}, C_{j}\right\}_{j=\alpha+1}^{\beta}$ be pairs and triples of pairwise disjoint sets such that for any $1 \leq i<j \leq \alpha+\beta$ we have $X_{i} \cap Y_{j} \neq \emptyset$ where $X$ and $Y$ can be any of $A, B$ and $C$. Then the following inequality holds:

$$
\begin{aligned}
\sum_{i=1}^{\alpha+\beta} \frac{2}{\left(\begin{array}{c}
\left|A_{i}\right|+\left|B_{i}\right| \\
\left|A_{i}\right|
\end{array}\right)}+\sum_{j=1}^{\beta} & \left(\frac{2}{\left(\begin{array}{c}
\left|A_{\alpha+j}\right|+\left|C_{\alpha+j}\right| \\
\left|A_{\alpha+j}\right|
\end{array}\right)}+\frac{2}{\left(\begin{array}{c}
\left.\left|B_{\alpha+j}\right|+\left|C_{\alpha+j}\right|\right) \\
\left|B_{\alpha+j}\right|
\end{array}\right)}\right. \\
& -\frac{2}{\left(\begin{array}{c}
\left|A_{\alpha+j}\right|+\left|B_{\alpha+j}\right|+\left|C_{\alpha+j}\right| \\
\left|A_{\alpha+j}\right|
\end{array}\right.}-\frac{2}{\left(\begin{array}{l}
\left|A_{\alpha+j}\right|+\left|B_{\alpha+j}\right|+\left|C_{\alpha+j}\right| \\
\left|B_{\alpha+j}\right|
\end{array}\right) \leq 1 .}
\end{aligned}
$$

Proof. Let us define $M$ to be $\bigcup_{i=1}^{\alpha}\left(A_{i} \cup B_{i}\right) \cup \bigcup_{j=1}^{\beta}\left(A_{\alpha+j} \cup B_{\alpha+j} \cup C_{\alpha+j}\right)$ and let us write $|M|=m$. Just as before, let us introduce a family $\left\{S_{i}, T_{i}\right\}_{i=1}^{2(\alpha+\beta)}$ of disjoint pairs as $S_{i}=A_{i}, T_{i}=B_{i}$ and $S_{2(\alpha+\beta)-j}=B_{j}, T_{2(\alpha+\beta)-j}=A_{j}$ for all $1 \leq i, j \leq$ $\alpha+\beta$. We count the pairs $(\pi, j)$ such that $\pi$ is a permutation of the elements of $M$ and $1 \leq j \leq 2(\alpha+\beta)$ with all elements of $S_{j}$ preceding all elements of $T_{j}$ in $\pi$ that is $\max \left\{\pi^{i-1}(s): s \in S_{j}\right\}<\min \left\{\pi^{-1}(t): t \in T_{j}\right\}$. We denote this by $S_{j}<_{\pi} T_{j}$. For every fixed $j$ there exist exactly $\left|S_{j}\right| !\left|T_{j}\right| !\left(m-\left|S_{j}\right|-\left|T_{j}\right|\right) !\left(\begin{array}{c}m \\ \left|S_{j}\right|+\left|T_{j}\right|\end{array}\right)$ permutations $\pi$ with $S_{j}<_{\pi} T_{j}$. On the other hand for any fixed $\pi$ there exists at most one $j$ with $S_{j}<_{\pi} T_{j}$. Indeed, if $i \neq j, 2(\alpha+\beta)-j$, then both $S_{i}$ and $T_{i}$ meet both $S_{j}$ and $T_{j}$, while clearly if $S_{j}<_{\pi} T_{j}$, then $S_{2(\alpha+\beta)-j}=T_{j} \nless_{\pi} S_{j}=T_{2(\alpha+\beta)-j}$. These observations would yield Bollobás's original inequality, but we haven't used the existence of the $C_{j}$ 's. Observe that if $A_{j}<_{\pi} C_{j}, C_{j}<_{\pi} A_{j}, B_{j}<_{\pi} C_{j}$ or $C_{j}<_{\pi} B_{j}$, then again by the cross-intersecting property $(\pi, i)$ can be a pair counted only if $i=j$ or $i=2(\alpha+\beta)-j$ and at least one of $A_{i}<_{\pi} B_{i} \cup C_{i}, B_{i} \cup C_{i}<_{\pi} A_{i}, C_{i} \cup B_{i}<_{\pi} A_{i}, C_{i} \cup A_{i}<_{\pi} B_{i}$ holds. Counting $j$ and $2(\alpha+\beta)-j$ cases together this yields

$$
\begin{gathered}
\sum_{j=1}^{\alpha+\beta} 2\left|A_{j}\right| !\left|B_{j}\right| !\left(m-\left|A_{j}\right|-\left|B_{j}\right|\right) !\left(\begin{array}{c}
m \\
\left|A_{j}\right|+\left|B_{j}\right|
\end{array}\right) \\
\leq m !-\sum_{j=1}^{\alpha+\beta} 2\left[\left|A_{j}\right| !\left|C_{j}\right| !\left(m-\left|A_{j}\right|-\left|C_{j}\right|\right) !\left(\begin{array}{c}
m \\
\left|A_{j}\right|+\left|C_{j}\right|
\end{array}\right)\right. \\
\left.+\left|B_{j}\right| !\left|C_{j}\right| !\left(m-\left|C_{j}\right|-\left|B_{j}\right|\right) !\left(\begin{array}{c}
m \\
\left|C_{j}\right|+\left|B_{j}\right|
\end{array}\right)\right]
\end{gathered}
$$




$$
\begin{aligned}
& +\sum_{j=1}^{\beta} 2\left|A_{\alpha+j}\right| !\left(\left|B_{\alpha+j}\right|+\left|C_{\alpha+j}\right|\right) !\left(m-\left|A_{\alpha+j}\right|-\left|B_{\alpha+j}\right|\right. \\
& \left.-\left|C_{\alpha+j}\right|\right) !\left(\begin{array}{c}
m \\
\left|A_{\alpha+j}\right|+\left|B_{\alpha+j}\right|+\left|C_{\alpha+j}\right|
\end{array}\right) \\
& +\sum_{j=1}^{\beta} 2\left|B_{\alpha+j}\right| !\left(\left|A_{\alpha+j}\right|+\left|C_{\alpha+j}\right|\right) !\left(m-\left|A_{\alpha+j}\right|-\left|B_{\alpha+j}\right|\right. \\
& \left.-\left|C_{\alpha+j}\right|\right) !\left(\begin{array}{c}
m \\
\left|A_{\alpha+j}\right|+\left|B_{\alpha+j}\right|+\left|C_{\alpha+j}\right|
\end{array}\right)
\end{aligned}
$$

Dividing by $m$ ! and rearranging yields the statement of the lemma.

We apply Lemma 2.1 to the families $\mathcal{F}_{h-m_{2}+1}, \ldots, \mathcal{F}_{h}$ with $\beta=m_{3}$ and $\alpha=m_{2}-$ $m_{3}$. As all sets in the $\mathcal{F}_{i}$ 's are of size $k$ we obtain

$$
\frac{2\left(m_{2}-m_{3}\right)}{\left(\begin{array}{c}
2 k \\
k
\end{array}\right)}+\frac{6 m_{3}}{\left(\begin{array}{c}
2 k \\
k
\end{array}\right)}-\frac{6 m_{3}}{\left(\begin{array}{c}
3 k \\
k
\end{array}\right)} \leq 1 .
$$

As $\left(\begin{array}{c}3 k \\ k\end{array}\right) \geq 3\left(\begin{array}{c}2 k \\ k\end{array}\right)$ for $k \geq 3$, the left hand side of the above equation is greater than $\frac{2\left(m_{2}-m_{3}\right)}{\left(\begin{array}{c}2 k \\ k\end{array}\right)}+\frac{4 m_{3}}{\left(\begin{array}{c}2 k \\ k\end{array}\right)}=\frac{2\left(m_{2}+m_{3}\right)}{\left(\begin{array}{c}2 k \\ k\end{array}\right)}$. Therefore we obtain $m_{2}+m_{3} \leq \frac{1}{2}\left(\begin{array}{c}2 k \\ k\end{array}\right)=\left(\begin{array}{c}2 k-1 \\ k-1\end{array}\right)$. So for $n<4 k$ we have the bound

$$
\sum_{i=1}^{h}\left|\mathcal{F}_{i}\right| \leq h+m_{2}+m_{3} \leq\left(\begin{array}{c}
n-1 \\
k-1
\end{array}\right)-\left(\begin{array}{c}
n-k-1 \\
k-1
\end{array}\right)+1+\left(\begin{array}{c}
2 k-1 \\
k-1
\end{array}\right) .
$$

Suppose first that $n \geq 3 k$ holds. Plugging into (2.3) we obtain the upper bound $\left(\begin{array}{l}n-1 \\ k-1\end{array}\right)+1$. To get rid of the extra 1, we need to use the uniqueness part of the Hilton-Milner theorem [11] that we used to get our bound on $h$. It states that if $k \geq 4$ and an intersecting family $\mathcal{F} \subseteq\left(\begin{array}{c}{[n]} \\ k\end{array}\right)$ with $\cap_{F \in \mathcal{F}} F=\emptyset$ has size $\left(\begin{array}{c}n-1 \\ k-1\end{array}\right)-\left(\begin{array}{c}n-k-1 \\ k-1\end{array}\right)+\overline{1}$, then there exist $x \in[n]$ and $x \notin G \subseteq[n]$ such that $\mathcal{F}=\{G\} \cup\{F: x \in F, F \cap G \neq \emptyset\}$. Observe that for any $H \neq G$ with $x \notin H$ there exist lots of sets $F \in \mathcal{F}$ that are disjoint with $H$, so only sets $H^{\prime}$ that contain $x$ can be added to the $\mathcal{F}_{j}$ 's. But as all $\mathcal{F}_{j}$ 's consist of pairwise disjoint sets, such an $H^{\prime}$ can only be added to the $\mathcal{F}_{j}$ containing $G$. Also, at most one such set can be added as again this $\mathcal{F}_{j}$ consists of pairwise disjoint sets. We obtained that if $t \geq 2$ and $h=\left(\begin{array}{l}n-1 \\ k-1\end{array}\right)-\left(\begin{array}{c}n-k-1 \\ k-1\end{array}\right)+1$, then $\sum_{j=1}^{h}\left|\mathcal{F}_{j}\right| \leq\left(\begin{array}{c}n-1 \\ k-1\end{array}\right)-\left(\begin{array}{c}n-k-1 \\ k-1\end{array}\right)+2<\left(\begin{array}{l}n-1 \\ k-1\end{array}\right)$.

Next, we assume that $2 k+2 \leq n<3 k$. Then we have $t \leq 2$ and therefore the family $\mathcal{F}^{\prime}:=\cup_{i=1}^{h} \mathcal{F}_{i}$ has the property that for any $F \in \mathcal{F}^{\prime}$ there exists at most one other $G \in \mathcal{F}^{\prime}$ that is disjoint with $F$. Such families are called $(\leq 1)$-almost intersecting and Gerbner et al. [8] proved that whenever $2 k+2 \leq n$ holds, then any $(\leq 1)$-almost intersecting family $\mathcal{G} \subseteq\left(\begin{array}{c}{[n]} \\ k\end{array}\right)$ has size at most $\left(\begin{array}{c}n-1 \\ k-1\end{array}\right)$.

Finally, if $n=16, k=4$, then we need to bound $h+m_{2}+m_{3}+m_{4} \leq h+m_{2}+2 m_{3} \leq$ $h+2 m_{2}+3 m_{3}$. As $\left(\begin{array}{c}3 k \\ k\end{array}\right)=\left(\begin{array}{c}12 \\ 4\end{array}\right)>6\left(\begin{array}{l}8 \\ 4\end{array}\right)=\left(\begin{array}{c}2 k \\ k\end{array}\right)$, (2.2) implies $2 m_{2}+3 m_{3} \leq\left(\begin{array}{l}8 \\ 4\end{array}\right)$. Using the Hilton-Milner bound $h \leq\left(\begin{array}{l}n-1 \\ k-1\end{array}\right)-\left(\begin{array}{c}n-k-1 \\ k-1\end{array}\right)+1$ and plugging in $n=16$, we obtain $\sum_{i=1}^{h}\left|\mathcal{F}_{i}\right| \leq h+2 m_{2}+3 m_{3} \leq\left(\begin{array}{l}n-1 \\ k-1\end{array}\right)-\left(\begin{array}{c}11 \\ 3\end{array}\right)+1+\left(\begin{array}{l}8 \\ 4\end{array}\right)<\left(\begin{array}{l}n-1 \\ k-1\end{array}\right)$. This concludes the proof. 
Proof of Theorem 1.2. Theorem 1.4 shows that $K n_{n, k} \leq\left(\begin{array}{l}n-1 \\ k-1\end{array}\right)$ holds if $n \geq 2 k+2$. Observe that $\operatorname{diam}\left(K n_{n, k}\right) \leq 3$ if and only if $n \geq 2.5 k-0.5$ (see e.g. [16]). Also, Theorem 1.3 yields that if the diameter of a graph $G$ is at most 3 , then any independent set in $G$ is in general position. The largest independent sets in $K n_{n, k}$ correspond to stars, i.e. families $\mathcal{S}_{x}=\left\{H \in\left(\begin{array}{c}{[n]} \\ k\end{array}\right): x \in H\right\}$ for some $x \in[n]$. Therefore, $g p\left(K n_{n, k}\right) \geq\left(\begin{array}{c}n-1 \\ k-1\end{array}\right)$ holds provided $n \geq 2.5 k-0.5$.

If $2 k+2 \leq n<2.5 k-0.5$, then the upper bound of Theorem 1.4 is based on the result of Gerbner et al. [8] on $(\leq 1)$-almost intersecting families. Their result also states that the only $(\leq 1)$-almost intersecting families of size $\left(\begin{array}{l}n-1 \\ k-1\end{array}\right)$ are stars. But if $n<2.5 k-0.5$, then $\left\{H \in\left(\begin{array}{c}{[n]} \\ k\end{array}\right): 1 \in H\right\}$ is not in general position as shown by the following example: let $n=2 k+M$ with $1 \leq M<0.5 k-0.5$ and $F_{1}=[k], F_{2}=\{1,2, \ldots, k-M-1\}$ $\cup\{k+1, k+2, \ldots, k+M+1\}$. We claim that $d_{K n_{n, k}}\left(F_{1}, F_{2}\right) \geq 4$. Indeed, as $C:=$ $[n] \backslash\left(F_{1} \cup F_{2}\right)$ is of size $k-1$, we have $d_{K n_{n, k}}\left(F_{1}, F_{2}\right) \geq 3$. Suppose $G_{1}, G_{2}$ are $k$-subsets of $[n]$ with $F_{1} \cap G_{1}=G_{1} \cap G_{2}=\emptyset$. Let us define $\ell=\left|G_{1} \cap F_{2}\right|$. As $G_{1}$ is disjoint with $F_{1}$, so with $F_{1} \cap F_{2}$, we have $\ell \leq M+1$. Therefore $\left|C \cap G_{1}\right| \geq k-M-1$ must hold. As $G_{2}$ is disjoint with $G_{1}$, we obtain $\left|C \cap G_{2}\right| \leq M$, but as $\left|F_{1} \backslash F_{2}\right|=M+1$ and $2 M+1<k, G_{2}$ must meet $F_{2}$, so indeed $d_{K n_{n, k}}\left(F_{1}, F_{2}\right) \geq 4$ holds. On the other hand, for any $x \in F_{2} \backslash F_{1}$ and $y, z \in F_{1} \backslash F_{2}$, the sets $F_{1}, C \cup\{x\}, F_{2} \backslash\{x\} \cup\{y\}, C \cup\{z\}, F_{2}$ form a path of length 4 , therefore a geodesic with $1 \in F_{2} \backslash\{x\} \cup\{z\}$. This shows that $\left\{H \in\left(\begin{array}{c}{[n]} \\ k\end{array}\right): 1 \in H\right\}$ is not in general position. Therefore if $2 k+2 \leq n<2.5 k-0.5$ holds, then we have $g p\left(K n_{n, k}\right)<\left(\begin{array}{l}n-1 \\ k-1\end{array}\right)$.

Finally, let us consider the case $n=2 k+1$. Again, vertices corresponding to sets of stars are not in general position and all other independent sets have size smaller than $\left(\begin{array}{l}n-1 \\ k-1\end{array}\right)$. So suppose $F, F^{\prime}$ are disjoint sets in a family $\mathcal{F}$ corresponding to vertices in general position. Then by Theorem 1.3 , for any set $G \neq F, F^{\prime}$ in $\mathcal{F}$ we must have $d(G, F)=d\left(G, F^{\prime}\right)$. Observe that in $K n_{2 k+1, k}$ we have $d\left(H, H^{\prime}\right)=\min \left\{2\left(k-\left|H \cap H^{\prime}\right|\right)\right.$, $\left.2\left|H \cap H^{\prime}\right|+1\right\}$.

Let us first assume that $k=2 l+1$ is odd. Then by the above, for any $G \in \mathcal{F}$ we must have $|G \cap F|=\left|G \cap F^{\prime}\right|=l$ and the unique element $x \in[2 k+1] \backslash\left(F \cup F^{\prime}\right)$ must belong to $G$. Therefore, with the notation of the proof of Theorem 1.4 , we have $m_{2}=1$ and $h \leq\left(\begin{array}{l}n-1 \\ k-1\end{array}\right)-\left(\begin{array}{c}n-k-1 \\ k-1\end{array}\right)+1$ and thus $|\mathcal{F}| \leq\left(\begin{array}{c}n-1 \\ k-1\end{array}\right)-\left(\begin{array}{c}n-k-1 \\ k-1\end{array}\right)+2<\left(\begin{array}{l}n-1 \\ k-1\end{array}\right)$.

Let us assume that $k=2 l$ is even. Then by the above, for any $G \neq F, F^{\prime}$ in $\mathcal{F}$ we must have $|G \cap F|=\left|G \cap F^{\prime}\right|=l$ and thus $G \subseteq F \cup F^{\prime}$. If we take one set from each disjoint pair, we obtain a family $\mathcal{G} \subseteq\left(\begin{array}{c}{[2 k]} \\ k\end{array}\right)$ such that any pairwise intersection is of the same size. By Fisher's inequality, we obtain that the number $m_{2}$ of pairs is at most $2 k$. Moreover, as all sets of $\mathcal{F}$ are $k$-subsets of $[2 k]$, we must have $h \leq \frac{1}{2}\left(\begin{array}{c}2 k \\ k\end{array}\right)$. Therefore, we need to show $\frac{1}{2}\left(\begin{array}{c}2 k \\ k\end{array}\right)+2 k<\left(\begin{array}{c}2 k \\ k-1\end{array}\right)=\left(\begin{array}{c}2 k \\ k\end{array}\right) \frac{k}{k+1}$ which is equivalent to $\frac{2 k(2 k+2)}{k-1}<\left(\begin{array}{c}2 k \\ k\end{array}\right)$. This holds for $k \geq 4$.

\section{Concluding remarks}

First of all, it remains an open problem to determine $g p\left(K n_{n, k}\right)$ for $2 k+1 \leq n<$ $2.5 k-0.5$.

Let us finish this short note with two remarks. First observe that an $(\leq 1)$-almost intersecting family $\mathcal{F} \subseteq\left(\begin{array}{c}{[n]} \\ k\end{array}\right)$ corresponds to a subset $U$ of the vertices of $K n_{n, k}$ such that $K n_{n, k}[U]$ does not contain a path on three vertices. There have been recent developments 
$[1,9,15]$ in the general problem of finding the largest possible size of a subset $U$ of the vertices of $K n_{n, k}$ such that $K n_{n, k}[U]$ does not contain some fixed forbidden graph $F$. Note that independently of the host graph $G$, if a subset $S$ of the vertices of $G$ is in general position, then $G[S]$ cannot contain a path on three vertices as an induced subgraph. Returning to the Kneser graph $K n_{n, k}$ it would be interesting to address the induced version of the vertex Turán problems mentioned above.

There have been lots of applications and generalizations of Bollobás's inequality. Very recently O'Neill and Verstraëte [13] obtained Bollobás type results for $k$-tuples. Their condition to generalize disjoint pairs is completely different from the condition of Lemma 2.1. More importantly pairwise disjoint, cross-intersecting families were introduced by Rényi [14] as qualitatively independent partitions if the extra condition that $\cup_{F \in \mathcal{F}_{i}} F=[n]$ holds for all $1 \leq i \leq h$ is added, and the uniformity condition $|F|=k$ for all $F \in \cup_{i=1}^{h} \mathcal{F}_{i}$ is replaced by $\left|\mathcal{F}_{i}\right|=d$ for all $1 \leq i \leq h$. Gargano, Körner and Vaccaro proved [7] that for any fixed $d \geq 2$ as $n$ tends to infinity the maximum number of qualitatively independent $d$-partitions is $2^{\left(\frac{2}{d}-o(1)\right) n}$. Based on their construction, for any fixed $d$ one can obtain $2^{(2-o(1)) k}$ many pairwise disjoint cross-intersecting $d$-tuples of $k$-sets as $k$ tends to infinity.

\section{ORCID iD}

Balázs Patkós (D) https://orcid.org/0000-0002-1651-2487

\section{References}

[1] M. Alishahi and A. Taherkhani, Extremal $G$-free induced subgraphs of Kneser graphs, J. Comb. Theory Ser. A 159 (2018), 269-282, doi:10.1016/j.jcta.2018.06.010.

[2] B. S. Anand, U. Chandran S. V., M. Changat, S. Klavžar and E. J. Thomas, Characterization of general position sets and its applications to cographs and bipartite graphs, Appl. Math. Comput. 359 (2019), 84-89, doi:10.1016/j.amc.2019.04.064.

[3] B. Bollobás, On generalized graphs, Acta Math. Acad. Sci. Hungar. 16 (1965), 447-452, doi: $10.1007 / \mathrm{bf01904851.}$

[4] S. V. Chandran and G. J. Parthasarathy, The geodesic irredundant sets in graphs, Int. J. Math. Comb. 4 (2016), 135-143, http: / / fs . unm. edu/ I JMC / I JMC-4-2016.pdf.

[5] P. Erdős, C. Ko and R. Rado, Intersection theorems for systems of finite sets, Quart. J. Math. Oxford 12 (1961), 313-320, doi:10.1093/qmath/12.1.313.

[6] V. Froese, I. Kanj, A. Nichterlein and R. Niedermeier, Finding points in general position, Internat. J. Comput. Geom. Appl. 27 (2017), 277-296, doi:10.1142/s021819591750008x.

[7] L. Gargano, J. Körner and U. Vaccaro, Sperner capacities, Graphs Combin. 9 (1993), 31-46, doi:10.1007/bf01195325.

[8] D. Gerbner, N. Lemons, C. Palmer, B. Patkós and V. Szécsi, Almost intersecting families of sets, SIAM J. Discrete Math. 26 (2012), 1657-1669, doi:10.1137/120878744.

[9] D. Gerbner, A. Methuku, D. T. Nagy, B. Patkós and M. Vizer, Stability results for vertex Turán problems in Kneser graphs, Electron. J. Combin. 26 (2019), \#P2.13 (12 pages), doi:10.37236/ 8130 .

[10] M. Ghorbani, H. R. Maimani, M. Momeni, F. R. Mahid, S. Klavžar and G. Rus, The general position problem on kneser graphs and on some graph operations, Discuss. Math. Graph Theory (2020), doi:10.7151/dmgt.2269. 
[11] A. J. W. Hilton and E. C. Milner, Some intersection theorems for systems of finite sets, Quart. J. Math. Oxford 18 (1967), 369-384, doi:10.1093/qmath/18.1.369.

[12] P. Manuel and S. Klavžar, A general position problem in graph theory, Bull. Aust. Math. Soc. 98 (2018), 177-187, doi:10.1017/s0004972718000473.

[13] J. O'Neill and J. Verstraete, Bollobás-type inequalities on set $k$-tuples, 2018, arXiv:1812.00537v1 [math.CO].

[14] A. Rényi, Foundations of Probability, Wiley, 1971.

[15] A. Taherkhani, Size and structure of large $(s, t)$-union intersecting families, 2019, arXiv:1903.02614 [math.CO].

[16] M. Valencia-Pabon and J.-C. Vera, On the diameter of Kneser graphs, Discrete Math. 305 (2005), 383-385, doi:10.1016/j.disc.2005.10.001. 